\title{
Functional transcriptomic annotation and protein-protein interaction network analysis identify NEK2, BIRC5, and TOP2A as potential targets in obese patients with luminal $A$ breast cancer
}

\author{
Miriam Nuncia-Cantarero ${ }^{1}$ (1) $\cdot$ Sandra Martinez-Canales ${ }^{2} \cdot$ Fernando Andrés-Pretel $^{2} \cdot$ Gabriel Santpere $^{3}$. \\ Alberto Ocaña ${ }^{2}$ (i) Eva Maria Galan-Moya ${ }^{1}$ (i)
}

Received: 18 October 2017 / Accepted: 29 December 2017 / Published online: 12 January 2018

(c) The Author(s) 2018. This article is an open access publication

\begin{abstract}
Purpose Although obesity is a risk factor for breast cancer, little effort has been made in the identification of druggable molecular alterations in obese-breast cancer patients. Tumors are controlled by their surrounding microenvironment, in which the adipose tissue is a main component. In this work, we intended to describe molecular alterations at a transcriptomic and protein-protein interaction (PPI) level between obese and non-obese patients.

Methods and results Gene expression data of 269 primary breast tumors were compared between normal-weight (BMI $<25$, $n=130$ ) and obese (IMC $>30, n=139$ ) patients. No significant differences were found for the global breast cancer population. However, within the luminal A subtype, upregulation of 81 genes was observed in the obese group (FC $\geq 1.4$ ). Next, we explored the association of these genes with patient outcome, observing that 39 were linked with detrimental outcome. Their PPI map formed highly compact cluster and functional annotation analyses showed that cell cycle, cell proliferation, cell differentiation, and cellular response to extracellular stimuli were the more altered functions. Combined analyses of genes within the described functions are correlated with poor outcome. PPI network analyses for each function were to search for druggable opportunities. We identified 16 potentially druggable candidates. Among them, NEK2, BIRC5, and TOP2A were also found to be amplified in breast cancer, suggesting that they could act as strategic players in the obese-deregulated transcriptome.

Conclusion In summary, our in silico analysis describes molecular alterations of luminal A tumors and proposes a druggable PPI network in obese patients with potential for translation to the clinical practice.
\end{abstract}

Keywords Breast cancer $\cdot$ Transcriptomic analysis $\cdot$ Protein-protein interaction $\cdot$ Clinical outcome $\cdot$ Novel druggable targets $\cdot$ Targeted therapy

Electronic supplementary material The online version of this article (https://doi.org/10.1007/s10549-017-4652-3) contains supplementary material, which is available to authorized users.

Eva Maria Galan-Moya

EvaMaria.Galan@uclm.es

1 Translational Oncology Laboratory, Centro Regional de Investigaciones Biomédicas (CRIB), Universidad de Castilla La Mancha (UCLM), C/Almansa 14, 02008 Albacete, Spain

2 Translational Research Unit, University Hospital, Albacete, Spain

3 Department of Neuroscience, Yale School of Medicine, New Haven, CT, USA

\section{Introduction}

Breast cancer is the leading cause of cancer-related death among women worldwide [1]. To this regard, several factors are involved in the initiation and promotion of breast tumors including molecular alterations at the genomic level such as mutations or copy number alterations [2, 3]. Indeed, using functional studies, some of these genomic modifications have been clearly associated with a malignant phenotype, contributing to the oncogenesis of epithelial cells $[4,5]$. In addition to these molecular alterations, cancer cells rely on the surrounding microenvironment, where non-transformed cells and stromal components facilitate tumor growth by the secretion of autocrine signals like growth factors [6]. Stimulation of cancer cells by paracrine-secreted factors 
from interstitial cells including fibroblasts, neutrophils, or endothelial cells can stimulate functions such as proliferation, survival, or migration, which are necessary to the tumor formation and dissemination [7, 8]. However, components of the tumor stroma depend on different conditions and can differ among individuals. Of note, adipose tissue is one of the main components of the breast cancer microenvironment, and therefore, accumulation of fat tissue in the stroma can modify settings of tumor cells and influence their survival [8]. As an example, increased presence of insulin or insulinlike growth factors can affect tumor growth but also response to treatment [9]. In this context, breast tumors that express estrogen receptors are more dependent on stimulating factors [10].

Besides being a risk factor for cancer, obesity has also been associated with detrimental patient outcome, especially in postmenopausal patients [5, 4]. A number of epidemiological studies have demonstrated that how obesity is directly related to cancer mortality. In this sense, an increased body mass index (BMI) has been strongly linked with poor survival in postmenopausic patients carrying estrogen receptor positive tumors [11]. One of the mechanisms proposed to explain how obesity increases breast cancer risk is that adipocyte-secreted hormones could be promoting tumor progression through an increase of cellular proliferation [12]. However, little effort has been put into clarifying how the excess of adipose tissue in the tumor niche influences the molecular characteristics of the residing malignant cells.

In the present article, we aimed to evaluate biological functions that differentiate breast cancer tumors from obese and non-obese patients. To do so, we performed transcriptomic followed by protein-protein interaction network analyses to recognize relevant biological functions with druggable implications.

\section{Materials and methods}

\section{Transcriptomic and gene expression analyses using bioconductor}

We used a public data set (GEO Data Set accession number: GSE 78958) to compare mRNA levels from 405 breast cancer tumors. Affymetrix CEL files were downloaded and analyzed with R 3.3.2 (Bioconductor package). Data from patients not matching our BMI criteria (BMI $<25$ or $\geq 30$ ) were excluded from the analysis, what reduced tumor samples to 269 . Before proceeding with the comparative analysis, we performed a statistical quality control (QC), including relative log expression (RLE) and normalize unscaled standard error (NUSE) graphs. QC validated all samples for the following comparative analysis. Normalization was performed using the robust multi-array (RMA) system, included in the affymetrix package, and screening with the genefilter package. Data comparison was done using the limma package, comparing array data from each patient group (normal weight: $\mathrm{BMI}<25$; obese: $\mathrm{BMI} \geq 30$ ). Once the matrix for the experimental design was established, we used the function $\mathrm{lmFit}$ to perform a linear adjustment and create the contrast matrix in agreement with the compared groups, necessary to accomplish the Bayesian adjustment to determine the final fold change.

\section{Analysis of patient characteristics}

Comparison of proportions between normal-weight and obese groups we performed for each variable including grade, TNM stage, and patient ethnicity. This comparison was fulfilled using either Pearson's Chi-square or Fisher's exact test; $* p<0.05, * * p<0.001$.

\section{Construction and analysis of PPI networks and functional annotation}

We used the online tool STRING (http://www.string-db. org) to construct interactome maps of deregulated genes (STRING v10 data accessed: 14/02/17 and 10/07/17). Thus, we constructed a PPI map for the underexpressed genes and another for the overexpressed genes. The indicated network properties include:

Nodes: number of proteins in the network; Edges: number of interactions; Node degree: average number of interactions; Clustering coefficient: indicates the tendency of the network to form clusters. The closer the local clustering coefficient is to 1 , the more likely it is for the network to form clusters; PPI enrichment $p$ value: indicates the statistical significance. Proteins are considered hubs when they have more interactions than the average $\left(n^{\circ}\right.$ interactions $>$ node degree).

Functional screening for overexpressed genes was performed using Ensembl database (http://www.ensembl.org).

\section{Evaluation of clinical outcome}

The free-access Kaplan-Meier (KM) Plotter Online Tool (http://kmplot.com/analysis/) was used to investigate the relationship between gene expression levels and patient's clinical outcome in luminal A breast cancer. Only overexpressed genes significantly associated with detrimental outcome (Hazard Ratio $>1$ and $p$ value $\leq 0.05$ ) were used for subsequent analysis $(n=39)$. This tool was also used to determine relapse free survival (RFS) and overall survival (OS) in combined analyses of all genes included within cell cycle, cell differentiation, cell proliferation, and cellular response to extracellular stimuli functions. All the analyses were performed independently by two authors (MNC and 
SMC) and reviewed by a third author (EMGM). No discrepancies were observed.

\section{Identification of drug candidates}

We initially used data from The Drug Gene Interaction Database (DGIdb) http://dgidb.genome.wustl.edu/ to identify potentially druggable genes and their associated drugs among PPI hub proteins. Next, we used information from Genecards (www.genecards.org), which contains information from several databases, to manually select further druggable target among the PPI hub proteins of each function. Then, as described above, we used the STRING tool to build the druggable obese interactome.

\section{Molecular alteration identification}

We used data contained at cBioportal (www.cbioportal.org), Breast Invasive Carcinoma TCGA, $n=816$ [13], to identify potential copy number alterations (amplification or deletion) and the presence of mutations in the druggable genes.

\section{Results}

\section{Differential gene expression between normal-weight and obese breast cancer patients with luminal $A$ tumors}

We performed gene expression analyses in a cohort of 269 breast cancer patients based on their body mass index (BMI). The initial comparison between normal-weight $(n=130)$ and obese $(n=139)$ patients including all breast cancer tumors did not show statistical differences between both groups. Therefore, we decided to perform the analysis in each intrinsic tumor specific subtype (basal-like, HER2, luminal A, and luminal B). While no significant differences were found for the Basal-like, HER2, and luminal B subtypes, we identified 177 deregulated genes in the luminal A subtype (Table 1). Using a fold change of 1.4, we selected 96 and 81 genes that were underexpressed and overexpressed, respectively, in the obese group (Fig.1 and Table S1). Of note, the analysis of patient characteristics showed no significant differences between groups in relation with clinical stage or tumor grade. However, African American women displayed a significant higher proportion of obese women (Table 2).

Next, we constructed protein-protein interaction (PPI) maps of both identified groups and analyzed the potential functional modules within the networks. Notably, we found a higher number of interactions among proteins in the overexpressed PPI network (node degree: 16.2; clustering coefficient: 0.569) when compared with the underexpressed
Table 1 Gene expression comparison between normal-weight (B.M.I < 25) and obese (B.M.I $\geq 30$ ) breast cancer patients for each molecular subtype

\begin{tabular}{llll}
\hline Breast cancer patients (GSE 78958; $n=269)$ & \\
\hline Intrinsic subtypes groups & $\begin{array}{l}\text { Normal weight } \\
(\text { B.M.I }<25)\end{array}$ & $\begin{array}{l}\text { Obese } \\
(\text { B.M.I } \geq 30)\end{array}$ & $\begin{array}{l}\text { Deregu- } \\
\text { lated } \\
\text { genes }\end{array}$ \\
\hline Basal-like $(n=64)$ & $n=30$ & $n=34$ & NS \\
HER2-enriched $(n=27)$ & $n=15$ & $n=12$ & NS \\
Luminal A $(n=145)$ & $n=68$ & $n=77$ & 177 \\
Luminal B $(n=33)$ & $n=17$ & $n=16$ & NS \\
\hline
\end{tabular}

(node degree: 1.58; clustering coefficient: 0.398). Indeed, while proteins in the underexpressed map did not exhibit any cohesion, the overexpressed PPI network contained a marked cluster unit (Fig.1). Functional annotation analyses of the overexpressed genes identified 32 biological functions (Fig. S1 and Table S2).

\section{Identification of upregulated genes associated with worse outcome}

Next, we intended to identify the role of the 81 overexpressed genes in relation with patient outcome. We used data contained in the KM plotter online tool [14] that enclose information for more than 5000 breast cancer patients. We identified that 39 genes were significantly associated with detrimental patient outcome, including relapse free survival (RFS), overall survival (OS), or both (Fig. 2a, b), in the subgroup of luminal A breast tumors. To investigate how these genes are interacting among them, we constructed their PPI network. Notably, the PPI map of the proteins codified by the bad prognosis-associated genes formed a highly interconnected cluster (node degree: 30.1 ; clustering coefficient: 0.901) (Fig. 2a), suggesting that they could act as components of a protein complex with functional links.

\section{Functional annotation analyses of worse outcome}

We performed functional annotation analyses of the genes which predicted unfavorable outcome to identify biological functions that led to a high clustering coefficient [15]. In line with our previous result, many of these genes participated in multiple functions and were, therefore, included in more than one functional group (Table S3). We identified 12 biological functions (Fig. 2c), being cell cycle (33 genes), cell proliferation (19 genes), differentiation (12 genes), and cellular response to extracellular (EC) stimuli (12 genes) the most represented.

The combined analysis of genes contained in each of the functions was associated with detrimental RFS and 


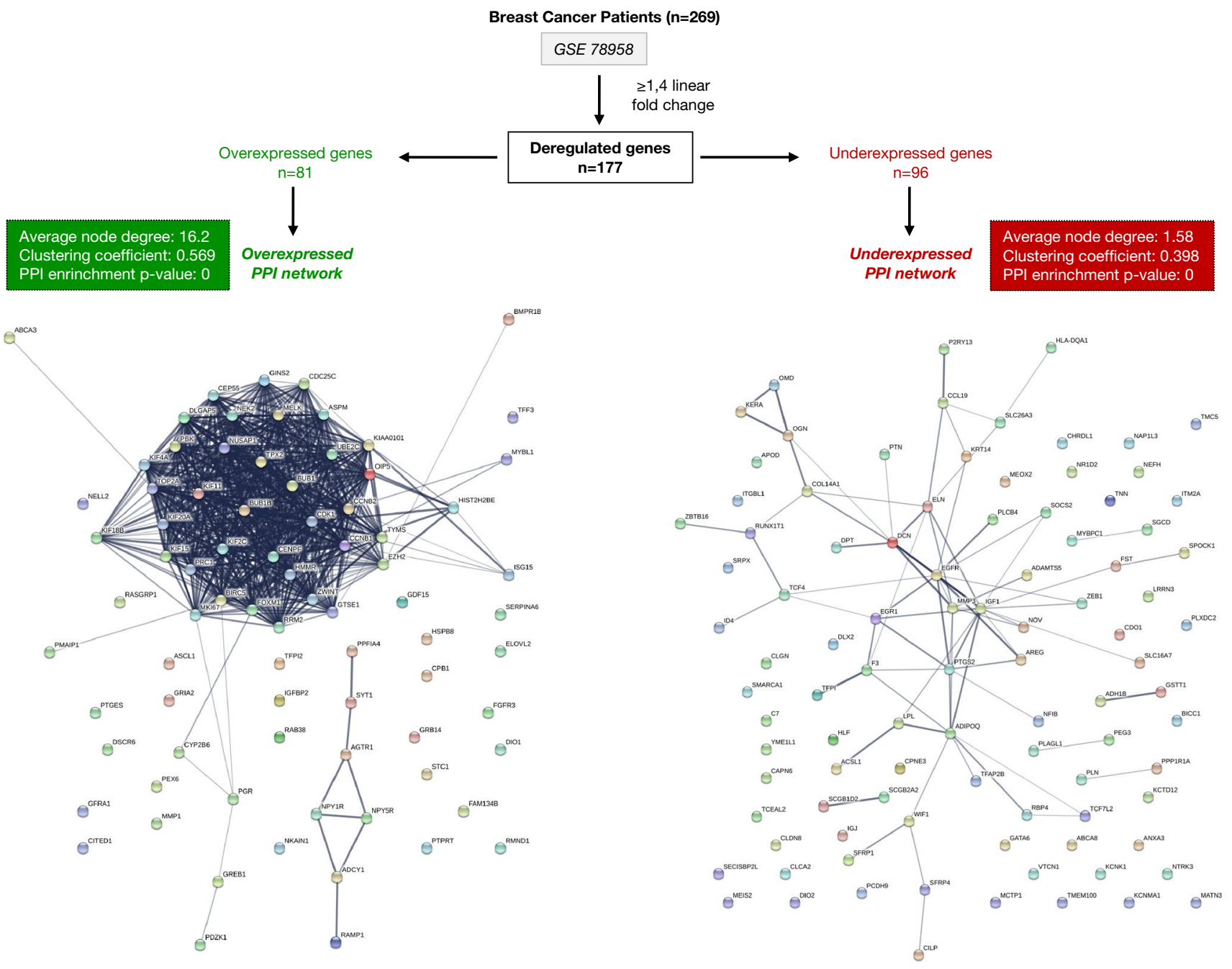

Fig. 1 Gene expression comparison between normal-weight and obese luminal A breast cancer patients. We identify 177 deregulated genes (fold change $\geq 1.4$ ) in luminal A obese patients. Then,

OS in luminal A (Figs. 3, 4). Cell cycle showed the best correlation with RFS [HR $=2.22(1.86-2.65), \log$ rank $p<1 \mathrm{e}-07]$ and a significant association with detrimental OS [HR $=2.71(1.85-3.97), \log$ rank $p=1 \mathrm{e}-07]$ (Fig. 3). Cell differentiation gene cluster predicts poor RFS $[H R=1.9(1.6-2.27), \log$ rank $p=3.4 \mathrm{e}-13]$ and OS $[\mathrm{HR}=2.36(1.62-3.43), \log$ rank $p=4.2 \mathrm{e}-06]$ (Fig. 3). Cell proliferation was also associated with lower RFS [HR $=2.12(1.78-2.53), \log$ rank $p<1 \mathrm{e}-16]$ and demonstrated the strongest association with poor OS $[\mathrm{HR}=2.73$ (1.86-4.01), log rank $p=9.4 \mathrm{e}-08]$ (Fig. 4). Finally, cellular response to EC stimuli gene showed poor RFS $[$ HR $=1.93(1.62-2.3), \log$ rank $p=1 \mathrm{e}-13]$ and OS $[\mathrm{HR}=2.34$ (1.61-3.4), log rank $p=4.6 \mathrm{e}-06]$ (Fig. 4). We also explored the potential of these functional groups to predict patient outcome in the other molecular subtypes. Of note, combined analysis of these functional genes using the online tool STRING, PPI networks for the underexpressed $(n=96)$ and the overexpressed $(n=81)$ genes were constructed

groups in Luminal B, HER2 and basal-like subtypes poorly or no significantly correlated with prognosis (Table S4).

\section{NEK2, BIRC5, and TOP2A are potential therapeutic targets in luminal $A$ obese patients}

Protein interactions can be used to prioritize gene candidates in in silico studies and to identify potential druggable targets [16]. PPI networks for these four functions confirmed their functional clustering unity (Fig. S2) and uncovered 18, 10, 6 , and 5 hub proteins for cell cycle, cell proliferation, cellular response to extracellular stimuli, and cell differentiation, respectively (Table S5). Of note, BUB1 and CDK1 were the components showing more interactions (edges).

Once we had identified core proteins for each functional cluster, we searched for druggable targets within the networks. Based on their interaction with existing drugs and/or 
Table 2 Proportion comparison of patients' clinical characteristics based on patients BMI

\begin{tabular}{llll}
\hline & $\%(n)$ & \multirow{2}{*}{$p$ value } \\
\cline { 2 - 3 } & $\begin{array}{l}\text { Normal } \\
\text { weight }<25 \\
(n=68)\end{array}$ & Obese $\geq 30(n=77)$ & \\
& & & \\
\hline Grade & & & \\
Well (Grade 1) & $50.9 \%(27)$ & $49.1 \%(26)$ & \\
Moderate (Grade & $48.7 \%(37)$ & $51.3 \%(39)$ & \\
$\quad$ 2) & & & \\
Poor (Grade 3) & $25.0 \%(4)$ & $75 \%(12)$ & \\
TNM Stage & & & \\
Stage I & $53.0 \%(35)$ & $47.0 \%(31)$ & \\
Stage II & $42.6 \%(26)$ & $57.4 \%(35)$ & \\
Stage III & $37.5 \%(6)$ & $62.5 \%(10)$ & \\
Stage IV & $50.0 \%(1)$ & $50.0 \%(1)$ & \\
Ethnicity & & & \\
African American & $30.0 \%(9)$ & $70.0 \%(21)$ & \\
European Ameri- & $48.6 \%(53)$ & $51.4 \%(56)$ & \\
$\quad$ can & & & \\
Other & $100.0 \%(6)$ & $0.0 \%(0)$ & \\
\hline
\end{tabular}

the existence of chemical inhibitors, we identified 16 potentially druggable candidates (Supplementary Table 6). We used the identified targetable proteins to construct a druggable PPI map (Fig. 5a). The resulting druggable network has a clustering coefficient tightly close to 1 (0.977), with 12 out of the 16 nodes showing interactions with all the network components (node degree $\geq 15$ ), what support the idea of a biological functional unit. Thus, the use of compounds against any node within the system might potentially affect the whole network, producing a wider response.

To complete our study, we searched for information about copy number alterations or mutations of the 16 identified druggable genes in the cancer genomics database (cBioportal, $[13,17]$. Deletions and mutations were present at a very low frequency. However, NIMA (Never in Mitosis Gene A)-Related Kinase 2 (NEK2), Apoptosis Inhibitor Survivin (BIRC5) and Topoisomerase (DNA) II Alpha (TOP2A) were found to be amplified in breast cancer $(12,6.3$, and 5.4\%, respectively) (Fig. 5b), suggesting a high potential role for them as therapeutic targets in luminal A patients.

\section{Discussion}

In the present article, we describe biological functions and PPI networks associated with obesity in luminal A tumors, which we found generally associated with worse outcome in luminal A patients. Moreover, we uncover a druggable PPI network on luminal A obese patients which could be of utility to design potential therapeutic strategies.
Although we initially investigated all four subtypes of breast cancer, we only found significant differences between normal-weight and obese patients for the luminal A subtype.

Although this might be due to the fact that luminal A group was the most abundant, the relevant association with outcome and the strong PPI network suggests a relevant biological role. Moreover, the combined analysis of genes within each function was associated with poor outcome in luminal A patients. Of note, the subgroup analysis did not correlate with patient outcome in the other three molecular subtypes (luminal B, HER2, and basal-like). This supports the idea that deregulation of these functions has a specific role in luminal A breast cancers. Our results are in line with those obtained in another study using the same transcriptomic database (GSE 78958) [18], although in this study, PPI analysis and druggable target opportunities were not explored. In addition, our study also identified a signature that is specific for luminal A tumors and explored the relation of this signature with patient outcome.

Although the level of deregulation between obese and non-obese patients was not highly elevated, we found significant differences using a fold changed of 1.4. We are aware that the fold change used is smaller than the one used in other studies [19, 20]. However, this finding could be explained by the fact that obese patients included in our study lack the exact BMI information. It could be expected that the exclusion of the obese-I subtype might have been of utility to increase the difference among our study groups. In any case, even the number of deregulated genes and the level was not high, the upregulation of these transcripts was associated with an important detrimental outcome.

The most frequent functions identified in the overexpressed genes and linked with worse outcome included: cell cycle, cell differentiation, cell proliferation, and cellular response to EC stimuli. Control of cell cycle was the most frequent pathway with genes involved in the formation of the mitotic spindle and centrosome or microtubules formation like BUB1, NUSAP, CENPF, CEP55, or the $K I F$ family [21]. In addition, other genes were associated with the regulation and control of the cell cycle such as $C D K$, GTSE1, CDC25C, or CCNB [22]. Finally, FOXM1, a transcription factor linked with the presence of a Luminal phenotype, was found to be upregulated [23]. Of note, our study also uncovered some interesting downregulated genes in the obese group. Notably, while EGFR is overexpressed in around 50\% TNBC and inflammatory breast cancers [24], we found that this gene, as well as its ligand $A R E G$, was downregulated in the luminal A obese group. Wnt signaling has been implicated in carcinogenesis as well as in obesity promotion $[25,26]$. In this line, luminal A obese group also showed a lower expression of Wnt pathway inhibitors, such as WIFl, BICCl, and the secreted 


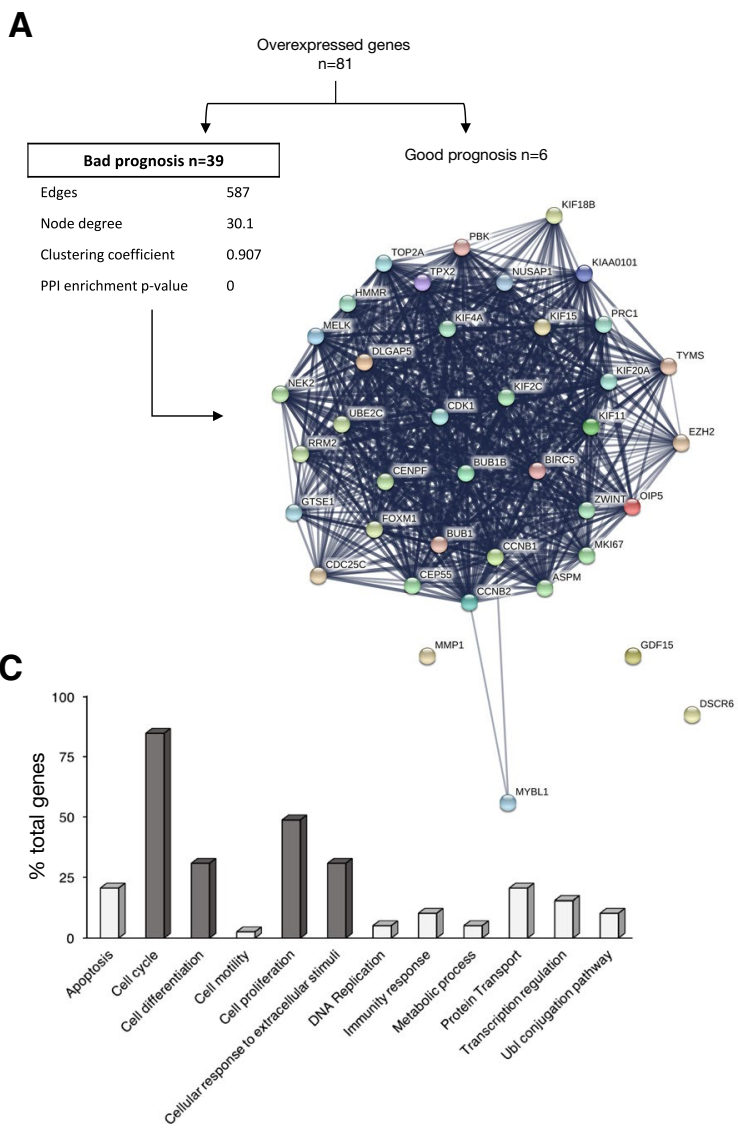

B

\begin{tabular}{|c|c|c|c|c|c|c|}
\hline \multirow[b]{3}{*}{ Gene } & & & \multicolumn{4}{|c|}{ KAPLAN MEIER PLOTTER } \\
\hline & \multicolumn{2}{|c|}{ ARRAY } & \multicolumn{2}{|l|}{ RFS } & \multicolumn{2}{|l|}{ os } \\
\hline & FC & $P$-value & HR & $P$-value & HR & $P$-Value \\
\hline $\begin{array}{l}\text { GDF15, Growth/differentiation factor } 15 \\
\end{array}$ & 1.801 & 0.000 & $1.3(1.1-1.54)$ & 0.003 & $1.45(1.02-2.07)$ & 0.040 \\
\hline MELK, Maternal embryonic leucine zipper kinase & 1.625 & 0.000 & $2.09(1.75-2.49)$ & 0.000 & $2.17(1.5-3.15)$ & 0.000 \\
\hline CEP55, Centrosomal protein of $55 \mathrm{kDa}$ & 1.620 & 0.000 & $2.21(1.85-2.65)$ & 0.000 & $2.48(1.71-3.62)$ & 0.000 \\
\hline RRM2, Ribonucleoside-diphosphate reductase subunit M2 & 1.591 & 0.000 & $2.09(1.75-2.49)$ & 0.000 & $2.1(1.45-3.04)$ & 0.000 \\
\hline KIF20A, Kinesin Family Member $20^{\mathrm{a}}$ & 1.585 & 0.000 & $1.81(1.52-2.15)$ & 0.000 & $2.2(1.52-3.18)$ & 0.000 \\
\hline TYMS, Thymidylate synthase & 1.575 & 0.000 & $1.72(1.44-2.04)$ & 0.000 & $2.01(1.4-2.9)$ & 0.000 \\
\hline TOP2A, DNA topoisomerase 2-alpha & 1.572 & 0.002 & $2.04(1.71-2.44)$ & 0.000 & $2.15(1.48-3.11)$ & 0.000 \\
\hline PRC1, Protein regulator of cytokinesis 1 & 1.547 & 0.000 & $2.28(1.9-2.73)$ & 0.000 & $2.56(1.75-3.74)$ & 0.000 \\
\hline CCNB2, G2/mitotic-specific cyclin-B2 & 1.525 & 0.000 & $2.29(1.9-2.75)$ & 0.000 & $2.73(1.86-4.01)$ & 0.000 \\
\hline KIF4A, Kinesin Family Member $4^{\mathrm{a}}$ & 1.522 & 0.000 & $1.96(1.64-2.33)$ & 0.000 & $2.4(1.65-3.48)$ & 0.000 \\
\hline ASPM, Abnormal spindle-like microcephaly-associated protein & 1.520 & 0.002 & $1.91(1.61-2.28)$ & 0.000 & $2.08(1.44-2.99)$ & 0.000 \\
\hline RIPPLY3, Protein ripply3 & 1.515 & 0.002 & $1.19(1-1.41)$ & 0.044 & $1.4(0.98-1.99)$ & 0.063 \\
\hline CDK1, Cyclin-dependent kinase 1 & 1.506 & 0.001 & $1.6(1.34-1.9)$ & 0.000 & $1.67(1.16-2.4)$ & 0.005 \\
\hline BIRC5, Baculoviral IAP repeat-containing protein 5 & 1.505 & 0.000 & $2.14(1.78-2.55)$ & 0.000 & $3.03(2.04-4.5)$ & 0.000 \\
\hline CENPF, Centromere protein $\mathrm{F}$ & 1.505 & 0.000 & $1.89(1.58-2.25)$ & 0.000 & $2.24(1.54-3.25)$ & 0.000 \\
\hline NEK2, Serine/threonine-protein kinase Nek2 & 1.495 & 0.001 & $1.97(1.65-2.35)$ & 0.000 & $2(1.39-2.89)$ & 0.000 \\
\hline TPX2, Protein TPX2 & 1.484 & 0.000 & $1.96(1.64-2.34)$ & 0.000 & $2.4(1.64-3.51)$ & 0.000 \\
\hline $\mathrm{CDC25C}, \mathrm{M}$-phase inducer phosphatase 3 & 1.479 & 0.000 & $1.54(1.29-1.82)$ & 0.000 & $1.9(1.32-2.74)$ & 0.001 \\
\hline HMMR, Hyaluronan mediated motility receptor & 1.479 & 0.001 & $1.72(1.45-2.06)$ & 0.000 & $2.01(1.39-2.88)$ & 0.000 \\
\hline OIP5, Protein Mis18-beta & 1.476 & 0.000 & $1.35(1.14-1.61)$ & 0.001 & $1.7(1.19-2.44)$ & 0.032 \\
\hline FOXM1, Forkhead box protein M1 & 1.461 & 0.000 & $1.88(1.58-2.25)$ & 0.000 & $2.86(1.94-4.23)$ & 0.000 \\
\hline BUB1, Mitotic checkpoint serine/threonine-protein kinase BUB1 & 1.461 & 0.000 & $2.12(1.78-2.53)$ & 0.000 & $2.42(1.67-3.51)$ & 0.000 \\
\hline DLGAP5, Disks large-associated protein 5 & 1.461 & 0.005 & $2.36(1.97-2.83)$ & 0.000 & $2.63(1.8-3.85)$ & 0.000 \\
\hline ISG15, Ubiquitin-like protein ISG15 & 1.460 & 0.007 & $1.43(1.2-1.7)$ & 0.000 & $1.34(0.94-1.91)$ & 0.109 \\
\hline NUSAP1, Nucleolar and spindle-associated protein 1 & 1.458 & 0.000 & $2.17(1.81-2.59)$ & 0.000 & $2.76(1.88-4.06)$ & .000 \\
\hline MK167, Proliferation marker protein Ki-67 & 1.457 & 0.000 & $1.92(1.61-2.29)$ & 0.000 & $2.66(1.81-3.89)$ & 0.000 \\
\hline KIAA0101, PCNA-associated factor & 1.449 & 0.001 & $1.92(1.61-2.29)$ & 0.000 & $1.92(1.33-2.77)$ & 0.000 \\
\hline ZWINT, ZW10 interactor & 1.445 & 0.000 & $1.83(1.53-2.18)$ & 0.000 & $1.57(1.1-2.24)$ & 0.012 \\
\hline PBK, PDZ Binding Kinase & 1.443 & 0.008 & $1.89(1.58-2.25)$ & 0.000 & $1.7(1.19-2.44)$ & 0.003 \\
\hline MMP1, Matrix Metallopeptidase 1 & 1.443 & 0.081 & $1.89(1.58-2.25)$ & 0.000 & $1.72(1.2-2.47)$ & 0.003 \\
\hline MYBL1, MYB Proto-Oncogene Like 1 & 1.443 & 0.011 & $1.47(1.23-1.74)$ & 0.000 & $1.45(1.02-2.07)$ & 0.039 \\
\hline UBE2C, Ubiquitin-conjugating enzyme E2 C & 1.441 & 0.000 & $1.81(1.51-2.15)$ & 0.000 & $1.98(1.37-2.86)$ & 0.000 \\
\hline $\begin{array}{l}\text { BUB1B, Mitotic checkpoint serine/threonine-protein kinase } \\
\text { BUB1 beta }\end{array}$ & 1.438 & 00 & 1) & 00 & 41) & 00 \\
\hline EZH2, Histone-lysine N-methyltransferase EZH2 & 1.437 & 0.000 & $1.57(1.32-1.86)$ & 0.000 & $19(1.51-3.16)$ & 0.000 \\
\hline GTSE1, G2 and S phase-expressed & 1.436 & 0.000 & $1.69(1.42-2.01)$ & 0.000 & $3.17(2.14-4.7)$ & 0.000 \\
\hline KIF 15 , Kinesin Family Member 15 & 1.434 & 0.000 & $1.63(1.37-1.94)$ & 0.000 & $2.14(1.48-3.08)$ & 0.000 \\
\hline CCNB1, G2/mitotic-specific cyclin-B & 1.431 & 0.000 & $1.73(1.45-2.05)$ & 0.000 & $1.86(1.29-2.68)$ & 001 \\
\hline KIF $11, \mathrm{~K}$ & 1.427 & 0.002 & & 0.000 & $2.22(1.53-3.2)$ & 0.000 \\
\hline KIF18B, Kinesin Family Member 18B & 1.421 & 0.000 & $1.51(1.27-1.79)$ & 0.000 & $2.56(1.76-3.72)$ & 0.000 \\
\hline
\end{tabular}

Fig. 2 PPI map and functional annotation of bad prognosis-associated upregulated genes in luminal A breast cancer obese patients. a Using the $\mathrm{K}-\mathrm{M}$ plotter tool, we identified overexpressed genes associated with bad prognosis and used them to construct a network of detrimental outcome in luminal A obese patients. b List of overexpressed genes associated with bad prognosis. Probe and transcript ID together with the symbol are indicated for each gene. Table includes the infor-

proteins $S F R P 1$ and $S F R P 4$, together with a downregulation of the negative regulators of MYC, TFAP2B, or TCF7L $[27,28]$.

Protein-protein interaction (PPI) networks offer information of how different proteins cooperate with others to trigger biological processes within the cell $[15,29]$. In this context, we have constructed PPI networks for the deregulated genes in luminal A obese patients. While proteins coded by deregulated genes poorly interact, we have found that exist a solid-clustering unit within the overexpressed PPI network. Remarkably, this dense cluster was comprised by proteins specifically coded by overexpressed genes that were associated with detrimental patient outcome. Thus, interference of one of its components might have an impact on several nodes, which could in turn lead to the destabilization of the network. This could open the window to new therapeutic strategies targeting this overexpressed PPI network in luminal A obese patients. mation for specific fold change difference, Hazard ratio (HR) for relapse free survival (RFS), and overall survival (OS). c Functional enrichment analyses of bad-prognosis-associated genes identifies cell cycle, cell differentiation, cell proliferation, and cellular response to extracellular stimuli as the most altered functions in luminal A obese patients

Next, we decided to search for potential drug targets within the PPI networks, linked with poor prognosis. Using Drug Interaction Database [30], we first identified eight druggable genes: BUB1, TOP2A, BIRC5, KIF11, NEK2, RRM2, TYMS, and PBK. Then, expanding the search to other drug databases, we added eight more druggable candidates: CCNB1, CDK1, FOXM1, KIF4A, KIF20A, MELK, NEK2, and CDC25C. The PPI network built with them exhibited a high degree of interactions and, as indicated by its high clustering coefficient, might act as a cohesive functional unit.

Mitotic-related targets in this druggable network are the aim of new chemical entities with potential for preclinical or clinical translation development [31]. For instance, a welldescribed target is TOP2A, where doxorubicin-like chemotherapies inhibit their effect $[32,33]$. Similarly, strategies to target BUB1 are under preclinical evaluation as this kinase has been described as associated with detrimental prognosis 

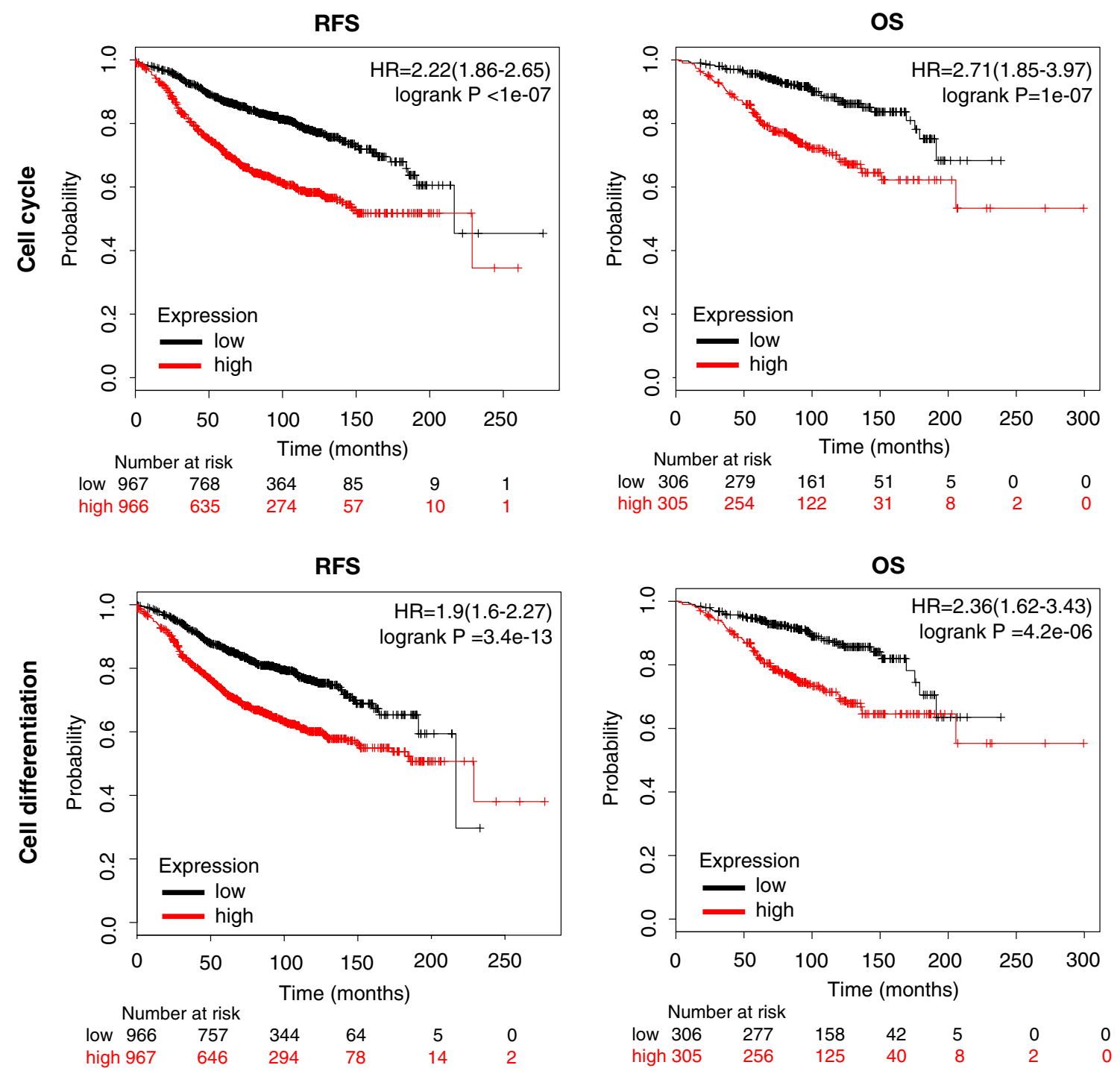

Fig. 3 Association with relapse free survival (RFS) and overall survival (OS) of gene sets included in the cell cycle and cell differentiation functions in luminal A breast cancer

in breast and ovarian cancer [20,34]. Compounds against KIF11 are under development [35] and some in clinical development [36].

BIRC5 codifies for a protein which is vital for the growth and survival of cancer cells. Survivin is found to be essential for several functions linked with oncogenic transformation [37]. It is known that normal tissues do not express survivin, and high expression in tumors is an indicative of poor prognosis and intrinsic resistance to radio- and chemotherapy [38]. As obese patients express high levels of BIRC5, evaluation of BIRC5 inhibitors, alone or in combination, could be a potential option.

Finally, NEK2 codifies for a serine-threonine kinase with a key role in mitosis that has been found to be aberrantly overexpressed in several cancer types, among them breast cancer [39, 40]. NEK2 expression levels are associated with tumor progression and detrimental outcome, as well as with drug resistance [41]. Besides, preclinical studies have shown that high NEK2 levels can induce tumorigenesis by mediating chromosome instability and aneuploidy, while its downregulation can lead to cancer cells death [42], suggesting a role for NEK2 as a potential target to treat cancer. Its elevated levels in obese patients points at NEK2 as a good candidate for targeted therapy in these patients. However, although several inhibitors have been developed for NEK2, some of them being highly specific and showing an irreversible inhibition, they have not been taken to clinical evaluation yet [43].

Notably, NEK2 as well as BIRC5 and TOP2A were amplified in more than 12,6 , and $5 \%$ of breast cancers, respectively, reinforcing their potential role as key therapeutic targets. 

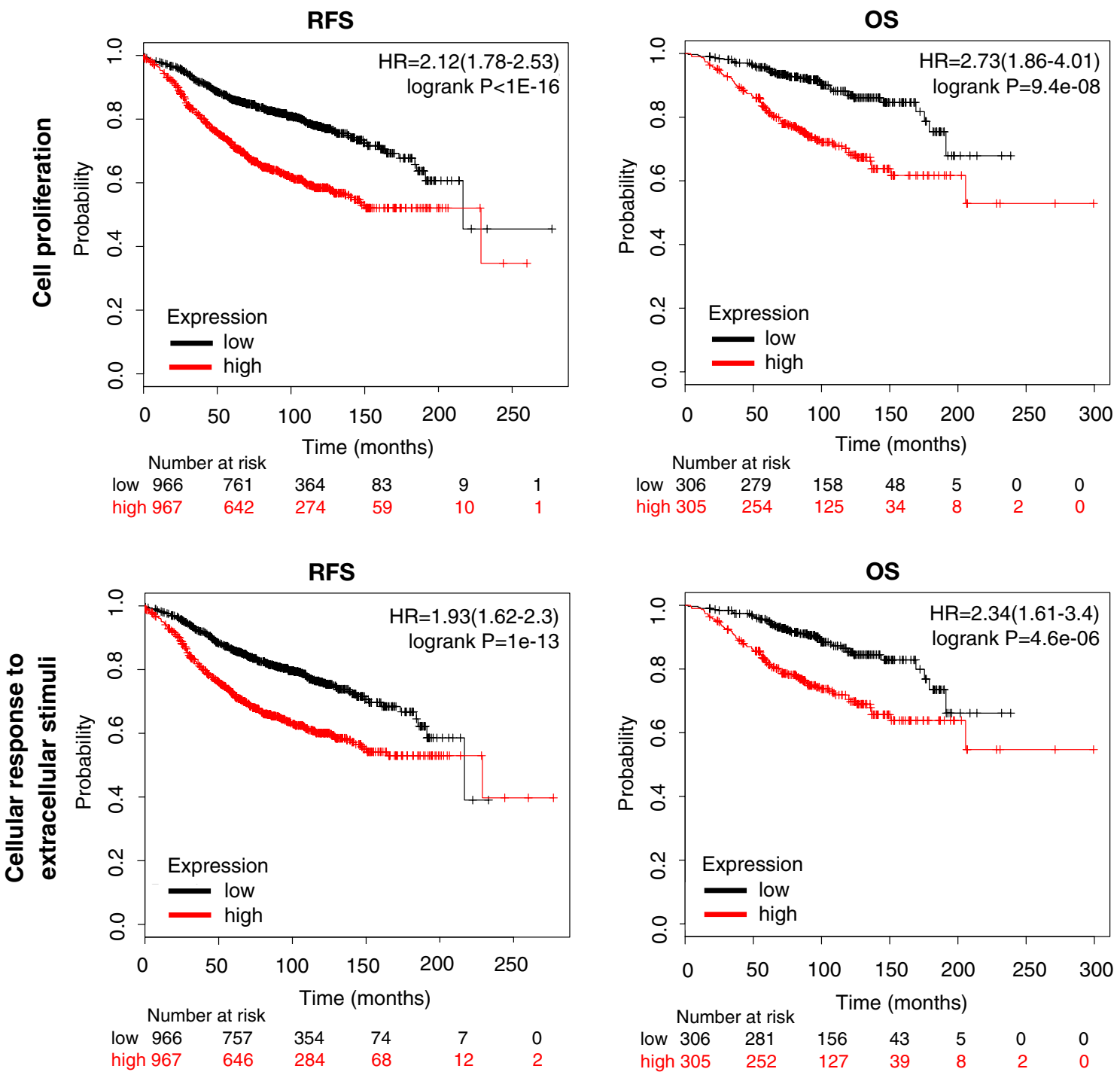

Fig. 4 Association with relapse free survival (RFS) and overall survival (OS) of gene sets included in the cell proliferation and cellular response to extracellular stimuli functions in luminal A breast cancer

In conclusion, in the present work, we describe functional pathways and protein-protein interacting networks associated with clinical outcome in luminal A tumors from obese patients. Moreover, we identify a druggable interacting map with potential for target inhibition. Although we acknowledge that this is an in silico analyses, and data should be confirmed in samples from patients, our results open new venues for further characterization and have potential for translation into the clinical setting. 


\section{A}

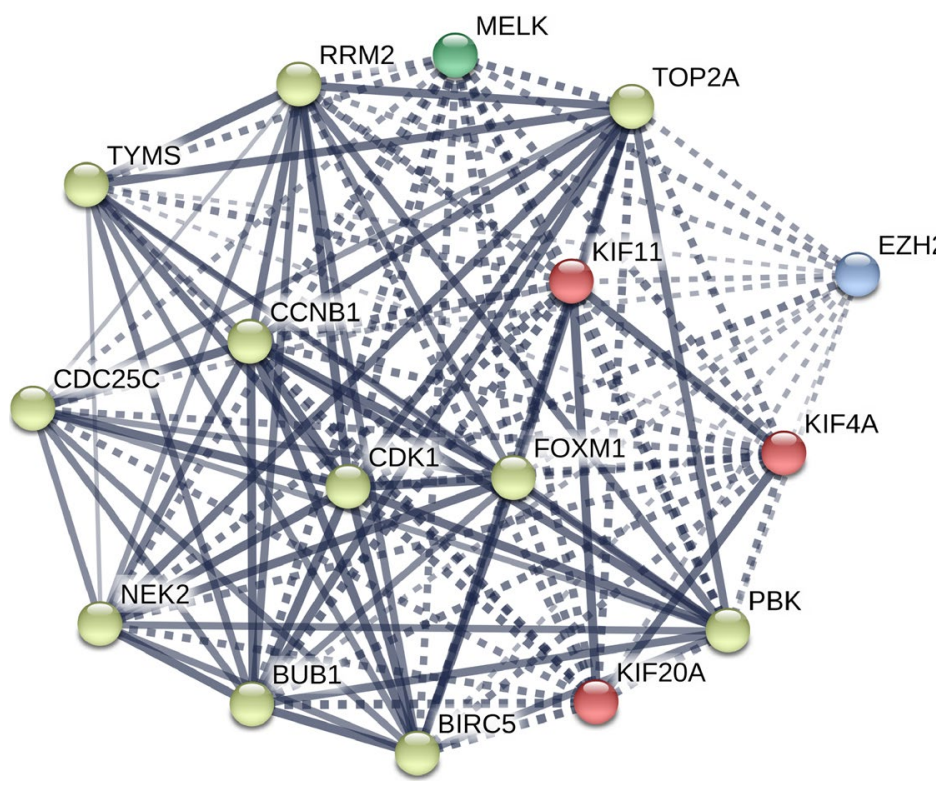

\begin{tabular}{lr}
\hline \multicolumn{2}{c}{ Druggable PPI Network } \\
\hline Nodes & 16 \\
\hline Edges & 117 \\
\hline Node degree & 14.6 \\
\hline Clustering coefficient & 0.977 \\
\hline PPI enrichment p-value & 0 \\
\hline
\end{tabular}

B

\begin{tabular}{lcccc} 
& & \multicolumn{2}{c}{ Breast Invasive Carcinoma (TCGA) } \\
\cline { 3 - 5 } BIRC5 & Edges $(n)$ & Amplification & Deletion & Mutation \\
\cline { 3 - 5 } BUB1 & $\mathbf{1 5}$ & $\mathbf{6 , 3} \%$ & $\mathbf{0 , 1} \%$ & - \\
CCNB1 & 15 & $0,5 \%$ & - & $0,4 \%$ \\
CDK1 & 15 & $0,1 \%$ & $1,3 \%$ & $0,5 \%$ \\
FOXM1 & 15 & $2 \%$ & $0,2 \%$ & $0,2 \%$ \\
KIF11 & 15 & $2,8 \%$ & $0,1 \%$ & $0,5 \%$ \\
KIF20A & 15 & $0,1 \%$ & $0,2 \%$ & $0,5 \%$ \\
KIF4A & 15 & $0,4 \%$ & $0,1 \%$ & $0,5 \%$ \\
MELK & 15 & - & $0,5 \%$ & $1,3 \%$ \\
PBK & 15 & $1,3 \%$ & $0,1 \%$ & $0,2 \%$ \\
RRM2 & 15 & $0,5 \%$ & $5,4 \%$ & $0,2 \%$ \\
TOP2A & 15 & $1,3 \%$ & $0,5 \%$ & $0,4 \%$ \\
NEK2 & 15 & $\mathbf{5 , 4 \%}$ & $\mathbf{0 , 5 \%}$ & $\mathbf{0 , 2} \%$ \\
TYMS & $\mathbf{1 4}$ & $\mathbf{1 2} \%$ & $\mathbf{0 , 1} \%$ \\
CDC25C & 14 & $0,9 \%$ & $0,1 \%$ & $0,1 \%$ \\
EZH2 & 13 & $0,4 \%$ & $0,1 \%$ & $0,5 \%$ \\
& 13 & $0,9 \%$ & $0,4 \%$
\end{tabular}

Fig. 5 Druggable PPI network in luminal A obese patients. a We used the online tool STRING to construct the PPI network of the druggable targets. b Percentage of copy number alterations (amplifi- cations and deletions), and presence of mutation for each druggable gene, obtained from cBioportal data 
Author contribution's EMGM conceived the project. AO and EMGM designed the study. All authors analyzed and interpreted data. AO and EMGM drafted and wrote the manuscript, and all authors revised it critically and contributed with intellectual content. All authors have approved the final version of this article. All authors agreed to be accountable for all aspects of the work.

Acknowledgements This work has been funded by Instituto de Salud Carlos III (PI16/01121), Diputación de Albacete and CRIS Cancer Foundation (to A. Ocaña) and the framework agreement between University of Castilla-La Mancha and Albacete Provincial Council (UCLM-Excma. Diputación de Albacete) in support to research activity (to E.M. Galan-Moya). We would like to also thanks to the cancer associations AMUMA and ACEPAIN for supporting part of this work. E.M. Galan-Moya is funded by the implementation research program of the UCLM (UCLM resolution date: 31/07/2014), with a contract for accessing the Spanish System of Science, Technology and InnovationSecti (co-funded by the European Commission/FSE funds).

\section{Compliance with ethical standards}

Conflict of interest The authors declare no potential conflict of interest in regard to the present article.

Open Access This article is distributed under the terms of the Creative Commons Attribution 4.0 International License (http://creativecomm ons.org/licenses/by/4.0/), which permits unrestricted use, distribution, and reproduction in any medium, provided you give appropriate credit to the original author(s) and the source, provide a link to the Creative Commons license, and indicate if changes were made.

\section{References}

1. Torre LA, Siegel RL, Ward EM, Jemal A (2016) Global cancer incidence and mortality rates and trends-an update. Cancer Epidemiol Prev Biomark 25:16-27. https://doi.org/10.1158/1055 -9965.EPI-15-0578

2. Beroukhim R, Mermel CH, Porter D et al (2010) The landscape of somatic copy-number alteration across human cancers. Nature 463:899-905. https://doi.org/10.1038/nature08822

3. Howell A, Anderson AS, Clarke RB et al (2014) Risk determination and prevention of breast cancer. Breast Cancer Res BCR 16:446. https://doi.org/10.1186/s13058-014-0446-2

4. van Gemert WA, Lanting CI, Goldbohm RA et al (2015) The proportion of postmenopausal breast cancer cases in the Netherlands attributable to lifestyle-related risk factors. Breast Cancer Res Treat 152:155-162. https://doi.org/10.1007/s10549-015-3447 $-7$

5. Crujeiras AB, Cabia B, Carreira MC et al (2016) Secreted factors derived from obese visceral adipose tissue regulate the expression of breast malignant transformation genes. Int J Obes 2005 40:514-523. https://doi.org/10.1038/ijo.2015.208

6. Bussard KM, Mutkus L, Stumpf K et al (2016) Tumor-associated stromal cells as key contributors to the tumor microenvironment. Breast Cancer Res BCR 18:84. https://doi.org/10.1186/s130 58-016-0740-2

7. Cirri P, Chiarugi P (2012) Cancer-associated-fibroblasts and tumour cells: a diabolic liaison driving cancer progression. Cancer Metastasis Rev 31:195-208. https://doi.org/10.1007/s10555-0119340-x
8. Mao Y, Keller ET, Garfield DH et al (2013) Stromal cells in tumor microenvironment and breast cancer. Cancer Metastasis Rev 32:303-315. https://doi.org/10.1007/s10555-012-9415-3

9. Denduluri SK, Idowu O, Wang Z et al (2015) Insulin-like growth factor (IGF) signaling in tumorigenesis and the development of cancer drug resistance. Genes Dis 2:13-25. https://doi. org/10.1016/j.gendis.2014.10.004

10. Strong AL, Strong TA, Rhodes LV et al (2013) Obesity associated alterations in the biology of adipose stem cells mediate enhanced tumorigenesis by estrogen dependent pathways. Breast Cancer Res BCR 15:R102. https://doi.org/10.1186/bcr3569

11. Turkoz FP, Solak M, Petekkaya I et al (2013) The prognostic impact of obesity on molecular subtypes of breast cancer in premenopausal women. J BUON Off J Balk Union Oncol 18:335-341

12. Booth A, Magnuson A, Fouts J, Foster M (2015) Adipose tissue, obesity and adipokines: role in cancer promotion. Horm Mol Biol Clin Investig 21:57-74. https://doi.org/10.1515/hmbci-2014-0037

13. Ciriello G, Gatza ML, Beck AH et al (2015) Comprehensive molecular portraits of invasive lobular breast cancer. Cell 163:506-519. https://doi.org/10.1016/j.cell.2015.09.033

14. Mihály Z, Kormos M, Lánczky A et al (2013) A meta-analysis of gene expression-based biomarkers predicting outcome after tamoxifen treatment in breast cancer. Breast Cancer Res Treat 140:219-232. https://doi.org/10.1007/s10549-013-2622-y

15. Pavlopoulos GA, Secrier M, Moschopoulos CN et al (2011) Using graph theory to analyze biological networks. BioData Min 4:10. https://doi.org/10.1186/1756-0381-4-10

16. Athanasios A, Charalampos V, Vasileios T, Ashraf GM (2016) Recent updates on the application of protein-protein interaction network in drug discovery. Curr Drug Metab

17. Cerami E, Gao J, Dogrusoz U et al (2012) The cBio cancer genomics portal: an open platform for exploring multidimensional cancer genomics data. Cancer Discov 2:401-404. https:// doi.org/10.1158/2159-8290.CD-12-0095

18. Toro AL, Costantino NS, Shriver CD et al (2016) Effect of obesity on molecular characteristics of invasive breast tumors: gene expression analysis in a large cohort of female patients. BMC Obes 3:22. https://doi.org/10.1186/s40608-016-0103-7

19. Pérez-Peña J, Serrano-Heras G, Montero JC et al (2016) In silico analysis guides selection of bet inhibitors for triple-negative breast cancer treatment. Mol Cancer Ther 15:1823-1833. http s://doi.org/10.1158/1535-7163.MCT-16-0004

20. Ocaña A, Pérez-Peña J, Alcaraz-Sanabria A et al (2016) In silico analyses identify gene-sets, associated with clinical outcome in ovarian cancer: role of mitotic kinases. Oncotarget 7:2286522872. https://doi.org/10.18632/oncotarget.8118

21. Appierto V, Tiberio P, Cavadini E et al (2009) Antimitotic effect of the retinoid 4-oxo-fenretinide through inhibition of tubulin polymerization: a novel mechanism of retinoid growthinhibitory activity. Mol Cancer Ther 8:3360-3368. https://doi. org/10.1158/1535-7163.MCT-09-0798

22. Sun X, Zhangyuan G, Shi L et al (2017) Prognostic and clinicopathological significance of cyclin B expression in patients with breast cancer: a meta-analysis. Medicine (Baltimore) 96:e6860. https://doi.org/10.1097/MD.0000000000006860

23. Rinaldetti S, Wirtz RM, Worst TS et al (2017) FOXM1 predicts overall and disease specific survival in muscle-invasive urothelial carcinoma and presents a differential expression between bladder cancer subtypes. Oncotarget. https://doi.org/10.18632/ oncotarget. 17394

24. Masuda H, Zhang D, Bartholomeusz C et al (2012) Role of epidermal growth factor receptor in breast cancer. Breast Cancer Res Treat 136:331-345. https://doi.org/10.1007/s10549-0122289-9

25. Xiao Q, Chen Z, Jin X et al (2017) The many postures of noncanonical Wnt signaling in development and diseases. Biomed 
Pharmacother Biomed Pharmacother 93:359-369. https://doi. org/10.1016/j.biopha.2017.06.061

26. Fuster JJ, Zuriaga MA, Ngo DT-M et al (2015) Noncanonical Wnt signaling promotes obesity-induced adipose tissue inflammation and metabolic dysfunction independent of adipose tissue expansion. Diabetes 64:1235-1248. https://doi.org/10.2337/db14-1164

27. Gauger KJ, Bassa LM, Henchey EM et al (2014) The effects of diet induced obesity on breast cancer associated pathways in mice deficient in SFRP1. Mol Cancer 13:117. https://doi.org/10.1186 /1476-4598-13-117

28. Ramakrishnan A-B, Cadigan KM (2017) Wnt target genes and where to find them. F1000Research 6:746. https://doi.org/10.1268 8/f1000research.11034.1

29. Safari-Alighiarloo N, Taghizadeh M, Rezaei-Tavirani M et al (2014) Protein-protein interaction networks (PPI) and complex diseases. Gastroenterol Hepatol Bed Bench 7:17-31

30. Wagner AH, Coffman AC, Ainscough BJ et al (2016) DGIdb 2.0: mining clinically relevant drug-gene interactions. Nucl Acids Res 44:D1036-1044. https://doi.org/10.1093/nar/gkv1165

31. Dominguez-Brauer C, Thu KL, Mason JM et al (2015) Targeting mitosis in cancer: emerging strategies. Mol Cell 60:524-536. http s://doi.org/10.1016/j.molcel.2015.11.006

32. Tubbs R, Barlow WE, Budd GT et al (2009) Outcome of patients with early-stage breast cancer treated with doxorubicin-based adjuvant chemotherapy as a function of HER 2 and TOP2A status. J Clin Oncol Off J Am Soc Clin Oncol 27:3881-3886. https://doi. org/10.1200/JCO.2008.20.1566

33. Bourdakou MM, Athanasiadis EI, Spyrou GM (2016) Discovering gene re-ranking efficiency and conserved gene-gene relationships derived from gene co-expression network analysis on breast cancer data. Sci Rep 6:20518. https://doi.org/10.1038/srep20518

34. Ocaña A, Pérez-Peña J, Díez-González L et al (2016) Transcriptomic analyses identify association between mitotic kinases, PDZbinding kinase and BUB1, and clinical outcome in breast cancer.
Breast Cancer Res Treat 156:1-8. https://doi.org/10.1007/s105 49-016-3720-4

35. Cross RA, McAinsh A (2014) Prime movers: the mechanochemistry of mitotic kinesins. Nat Rev Mol Cell Biol 15:257-271. http s://doi.org/10.1038/nrm3768

36. Rath O, Kozielski F (2012) Kinesins and cancer. Nat Rev Cancer 12:527-539. https://doi.org/10.1038/nrc3310

37. Groner B, Weiss A (2014) Targeting survivin in cancer: novel drug development approaches. BioDrugs Clin Immunother Biopharm Gene Ther 28:27-39. https://doi.org/10.1007/s40259-0130058-x

38. Hamy AS, Bieche I, Lehmann-Che J et al (2016) BIRC5 (survivin): a pejorative prognostic marker in stage II/III breast cancer with no response to neoadjuvant chemotherapy. Breast Cancer Res Treat 159:499-511. https://doi.org/10.1007/s10549-016-3961-2

39. Hayward DG, Clarke RB, Faragher AJ et al (2004) The centrosomal kinase Nek2 displays elevated levels of protein expression in human breast cancer. Cancer Res 64:7370-7376. https://doi. org/10.1158/0008-5472.CAN-04-0960

40. Tsunoda N, Kokuryo T, Oda K et al (2009) Nek2 as a novel molecular target for the treatment of breast carcinoma. Cancer Sci 100:111-116. https://doi.org/10.1111/j.1349-7006.2008.0100 7.x

41. Zhou W, Yang Y, Xia J et al (2013) NEK2 induces drug Resistance mainly through activation of efflux drug pumps and is associated with poor prognosis in myeloma and other cancers. Cancer Cell 23:48-62. https://doi.org/10.1016/j.ccr.2012.12.001

42. Cappello P, Blaser H, Gorrini C et al (2014) Role of Nek2 on centrosome duplication and aneuploidy in breast cancer cells. Oncogene 33:2375-2384. https://doi.org/10.1038/onc.2013.183

43. Fang Y, Zhang X (2016) Targeting NEK2 as a promising therapeutic approach for cancer treatment. Cell Cycle 15:895-907. http s://doi.org/10.1080/15384101.2016.1152430 